\section{(C) OPEN ACCESS}

\title{
Relationship between altitude and the prevalence of hypertension in Tibet: a systematic review
}

\author{
Cuomu Mingji, ${ }^{1}$ Igho J Onakpoya, ${ }^{2}$ Rafael Perera, ${ }^{2}$ Alison M Ward, ${ }^{2}$ Carl J Heneghan ${ }^{2}$
}

- Additional material is published online only. To view please visit the journal online (http://dx.doi.org/10.1136/ heartjnl-2014-307158).

${ }^{1}$ Tibetan Medical College, Lhasa, Tibet \& Austrian Academy of Sciences, Vienna, Austria

${ }^{2}$ Nuffield Department of Primary Care Health Sciences, Centre for Evidence-Based Medicine, University of Oxford, Oxford, UK

\section{Correspondence to} Dr Igho Onakpoya, Nuffield Department of Primary Care Health Sciences, Centre for Evidence-Based Medicine, University of Oxford, New Radcliffe House, Radcliffe Observatory Quarter, Oxford OX2 6GG, UK; igho.onakpoya@phc.ox.ac.uk.

Received 11 November 2014 Revised 31 March 2015 Accepted 7 April 2015 Published Online First 7 May 2015

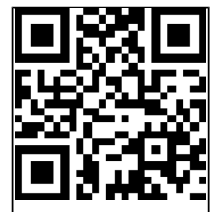

1054-1060.

\begin{abstract}
Introduction Hypertension is a leading cause of cardiovascular disease, which is the cause of one-third of global deaths and is a primary and rising contributor to the global disease burden. The objective of this systematic review was to determine the prevalence and awareness of hypertension among the inhabitants of Tibet and its association with altitude, using the data from published observational studies.
\end{abstract}

Methods We conducted electronic searches in Medline, Embase, ISI Web of Science and Global Health. No gender or language restrictions were imposed. We assessed the methodological characteristics of included studies using the Strengthening the Reporting of Observational Studies in Epidemiology (STROBE) criteria. Two reviewers independently determined the eligibility of studies, assessed the methodology of included studies and extracted the data. We used meta-regression to estimate the degree of change in hypertension prevalence with increasing altitude.

Results We identified 22 eligible articles of which eight cross-sectional studies with a total of 16913 participants were included. The prevalence of hypertension ranged between $23 \%$ and $56 \%$. A scatter plot of altitude against overall prevalence revealed a statistically significant correlation $(r=0.68 ; p=0.04)$. Meta-regression analysis revealed a $2 \%$ increase in the prevalence of hypertension with every $100 \mathrm{~m}$ increase in altitude $(p=0.06)$. The locations and socioeconomic status of subjects affected the awareness and subsequent treatment and control of hypertension. Conclusions The results from cross-sectional studies suggest that there is a significant correlation between altitude and the prevalence of hypertension among inhabitants of Tibet. The socioeconomic status of the inhabitants can influence awareness and management of hypertension. Very little research into hypertension has been conducted in other prefectures of Tibet where the altitude is much higher. Further research examining the impact of altitude on blood pressure is warranted.

\section{INTRODUCTION}

Hypertension is a leading cause of cardiovascular disease, which is the cause of one-third of global deaths and is a primary and rising contributor to the global disease burden. ${ }^{1}$

Exposure to hypoxia at high altitude is increasingly being recognised as a risk factor for hypertension. ${ }^{2} 3$ However, studies investigating the relationship between high altitude and hypertension are scarce. ${ }^{4}$ Because altitude-related hypertension is primarily related to hypoxia, gaining an insight into the hypoxia-hypertension mechanism may help with management of hypoxia-related conditions. This study focused on Central Tibet where there is concentration of inhabitants living in high plateau.

Tibet is known as the 'Third Pole' and is one of the highest inhabited areas of the world. The average altitude is $4500 \mathrm{~m}$ above sea level. Life expectancy is stated at 67 years old, ${ }^{5}$ which is lower than Inland China by 8 years. ${ }^{6}$ However, the leading causes of death in Tibet have not been identified due to an absence of data. In addition, there are no reliable statistical results to illustrate healthcare service coverage, and the general infrastructure such as the condition of roads is still very poor, with severely sick patients often dying on their way to the nearest clinic or hospital due to serious limitations in overall living conditions.

Data from observational and genetic studies have shown that Tibet has a higher hypertension rate than in other Chinese areas, ${ }^{78}$ as well as non-Chinese regions where inhabitants live at high altitudes. ${ }^{9}$ This leads to further serious comorbidities such as stroke, with a much higher prevalence than other Chinese areas. ${ }^{10}$ However, the health surveys on the prevalence of hypertension in Tibet are limited, as the currently available evidence only indicates data collected mainly from the City of Lhasa (the capital of Tibet) and nearby counties, while hardly any research has been conducted during the last three decades in other regions of Tibet, where there are higher altitudes and more harsh living conditions.

Until now, causative studies have mainly focused on two factors: the special environmental features of Tibet-the high altitude, and the relatively unique lifestyle led by indigenous people, looking specifically at diet. With the latter, significantly higher sodium levels were found among Tibetan subjects compared with other ethnic groups in China. ${ }^{11}$ Contradictory theories have been proposed regarding the mechanistic pathway of blood pressure (BP) regulation on exposure to high altitudes. Some authors have hypothesised that exposure to high altitude results in increased $\mathrm{BP}^{12}{ }^{13}$ possibly due to greater autonomic or sympathetic activities, ${ }^{14}$ while other investigators have postulated that the initial elevation observed on exposure to high altitude normalises and even drops below what is observed at sea level, after years of habitation at high altitudes. ${ }^{15-19}$ Evidence from previous research has also suggested an increased prevalence of hypertension at high altitudes, ${ }^{20}{ }^{21}$ possibly due to greater stimulation of the chemoreceptor reflex in individuals with borderline hypertension. ${ }^{14} 16$

Despite variable hypotheses around the correlation between high altitude and hypertension, there 
has not been a systematic review of the studies of hypertension in Tibet. This has resulted in the lack of a commonly identifiable conclusion on the correlation between high altitude and hypertension. Therefore, the objective of this systematic review is to determine the prevalence and awareness of hypertension among the inhabitants of Tibet and its association with altitude, using the data from published observational studies.

\section{METHODS}

We conducted electronic searches of the following databases: Medline, Embase, ISI Web of Science and Global Health. Each database was searched from inception until February 2014. Search terms used included blood pressure, hypertension, prevalence, altitude, Tibet and derivatives of these (a comprehensive Medline search strategy used has been included as an appendix) (see online supplementary appendix 1). We also searched the bibliographies of all located articles and no gender or language restrictions were imposed.

We included cross-sectional and observational studies. For inclusion, studies had to report the prevalence of hypertension among residents $\geq 15$ years old domiciled in any Tibetan province as an outcome. Studies not conducted in Central Tibet or Tibetan provinces of China were excluded from the review. We assessed the methodological characteristics of included studies using a checklist adapted from the Strengthening the Reporting of Observational Studies in Epidemiology (STROBE) criteria. $^{22}$ Two reviewers (CM and IJO) independently determined the eligibility of studies, assessed the methodology of included studies and extracted the data. Disagreements were resolved through consensus.

We extracted data according to study design, setting and altitude, participants' demographics, socioeconomic status, diagnostic criteria and the prevalence of hypertension, awareness, treatment and control rates of hypertension. Summary tables were used to report the methodological quality and characteristics of main results of included studies, while scatter plots were used to explore the relationship between altitude and the prevalence of hypertension. Meta-regression statistics using the Stata statistical package (V.13) was used to investigate the degree of change in the prevalence of hypertension with increasing altitude. We also performed meta-regression based on imputed mean age using either the one given or by imputation from studies with similar age range.

\section{RESULTS}

The initial search of data identified 286 potential articles (figure 1). After scanning the titles and abstracts, 22 articles were eligible for full textual review. Among these, six were

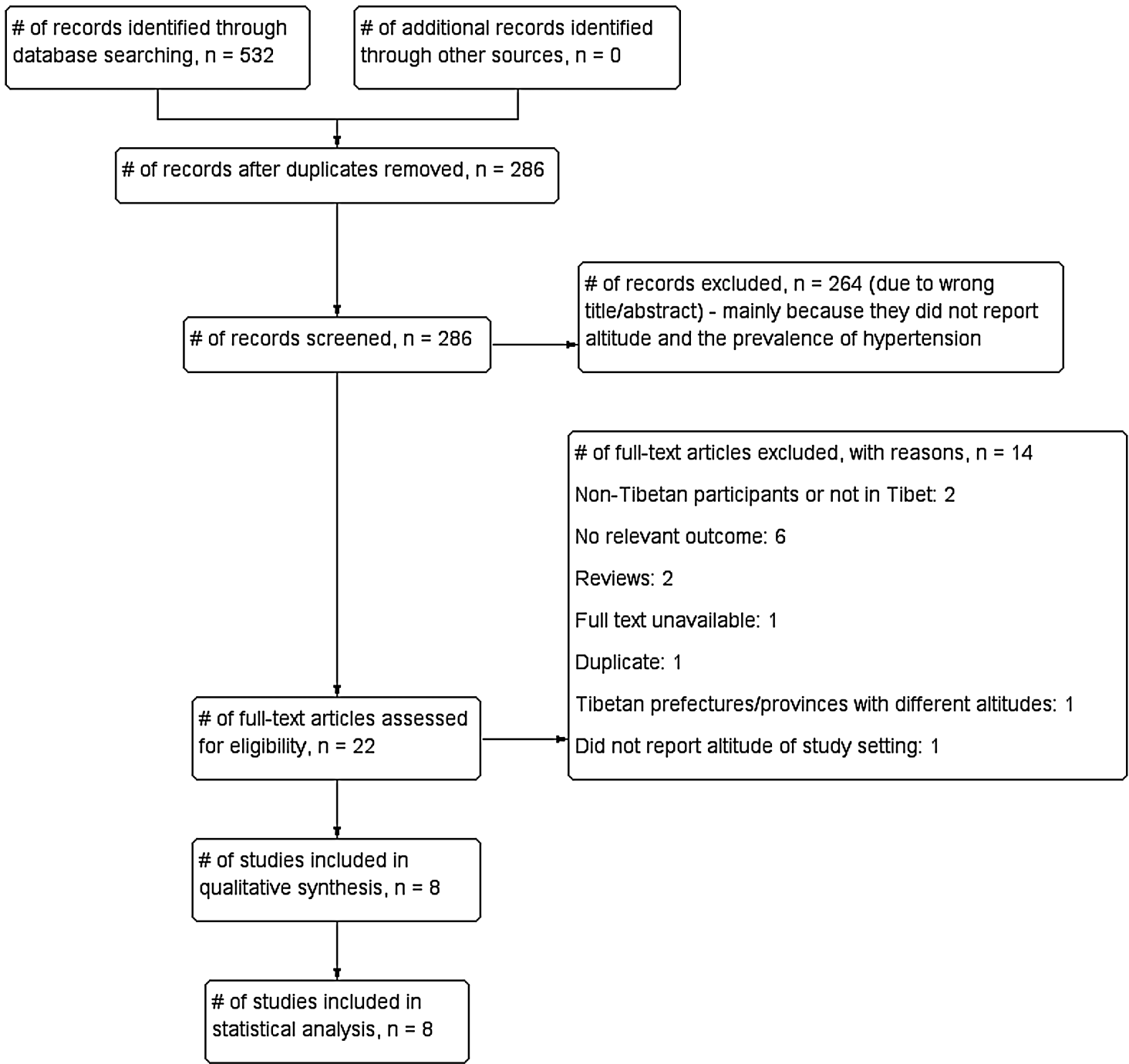

Figure 1 Flow diagram showing process for the inclusion of observational studies exploring the association between altitude and the prevalence of hypertension among Tibetan inhabitants. 
excluded due to having no relevant outcome; ${ }^{16}{ }^{23-26}$ two because they presented research which was either not conducted in Tibet ${ }^{27}$ or where the targeted subjects were non-Tibetans; ${ }^{28}$ two due to being review papers; ${ }^{10}{ }^{29}$ one because the full text could not be retrieved; $;^{30}$ one ${ }^{31}$ due to being a duplicate of another study included in the review; one ${ }^{7}$ because it was conducted across settings with different altitudes and one because it did not report the altitude of the study setting. ${ }^{32}$ This left eight studies with a total of 16913 participants for inclusion in the review. ${ }^{11}$ 33-39

There were some discrepancies in the reporting characteristics of the included studies (table 1). All eight were cross-sectional in design. Three used stratified sampling methods to recruit participants, ${ }^{33-35}$ three used simple randomised sampling ${ }^{11} 3638$ and one study used mixed methods for sampling. ${ }^{39}$ The sampling method used in one study was not specified. ${ }^{37}$ The sample size for respondents in all studies was representative of the defined populations; across studies, the response rates ranged between $65 \%$ and $100 \%$.

The definition of hypertension was consistent across seven studies ( $\geq 140 / 90 \mathrm{~mm} \mathrm{Hg}$ ); one study ${ }^{33}$ used $\geq 130 / 85 \mathrm{~mm} \mathrm{Hg}$ as cut-off criteria (table 1). Awareness was defined as self-report of prior diagnosis of hypertension by a healthcare professional (three studies) and self-report prior to the survey (two studies). Three of the included studies did not define the criteria for awareness. Five studies defined treatment of hypertension as selfreported use of medication prescribed by a healthcare professional, while three studies did not define hypertension treatment. Five of the included studies defined control of hypertension as medication use resulting in $\mathrm{BP}<140 / 90 \mathrm{~mm} \mathrm{Hg}$.

The settings varied between studies. Four studies were conducted using a mixture of rural and urban areas, ${ }^{34} 353839$ three in urban ${ }^{11} 36{ }^{37}$ and one exclusively in rural areas. ${ }^{33}$ The age of participants across the studies was between 15 and 90 years. Only three studies reported socioeconomic status; one of these was conducted in an urban area and included professionals, whereas the other studies included either herdsmen and/or farmers as participants (table 2).

The altitudes ranged from 3000 to $4300 \mathrm{~m}$, and the prevalence of hypertension ranged between $23 \%$ and $56 \%$ (table 2). A scatter plot of altitude against overall prevalence excluding this study (figure 2 ) showed a statistically significant correlation $(\mathrm{r}=0.68 ; \mathrm{p}=0.04)$. Meta-regression revealed that an increase in altitude of $1 \mathrm{~m}$ correlates with a $0.02 \%$ increase in prevalence $(\mathrm{p}=0.06$; figure 3$)$. Meta-regression based on imputed mean age using either the one given or imputing from a study that had similar age range did not reveal any observed change in the association (figure 4). However, there was insufficient data to explore the influence of age as a confounder.

Among the studies that used same standards of diagnostic criteria and had similar age range, the lowest hypertension prevalence was in Ganzi County, Sichuan Province, at 23\%, ${ }^{38}$ whereas the highest prevalence was at $56 \%$ in YangbajingDangxiong, Lhasa, which has the highest altitude at $4300 \mathrm{~m} .{ }^{34}$

Four studies were conducted in the City of Lhasa or the surrounding countryside administered under the Lhasa Municipality. ${ }^{11} 333536$ Though a scatter plot suggested a trend towards increased prevalence of hypertension with increasing altitude in these studies, the relationship did not achieve statistical significance $(r=0.65, p=0.17)$. Studies conducted in different Tibetan areas of Sichuan (Ganzi and Litang Counties) ${ }^{37} 38$ which had no marked differences in dietary habits also showed similar trends (data not shown).

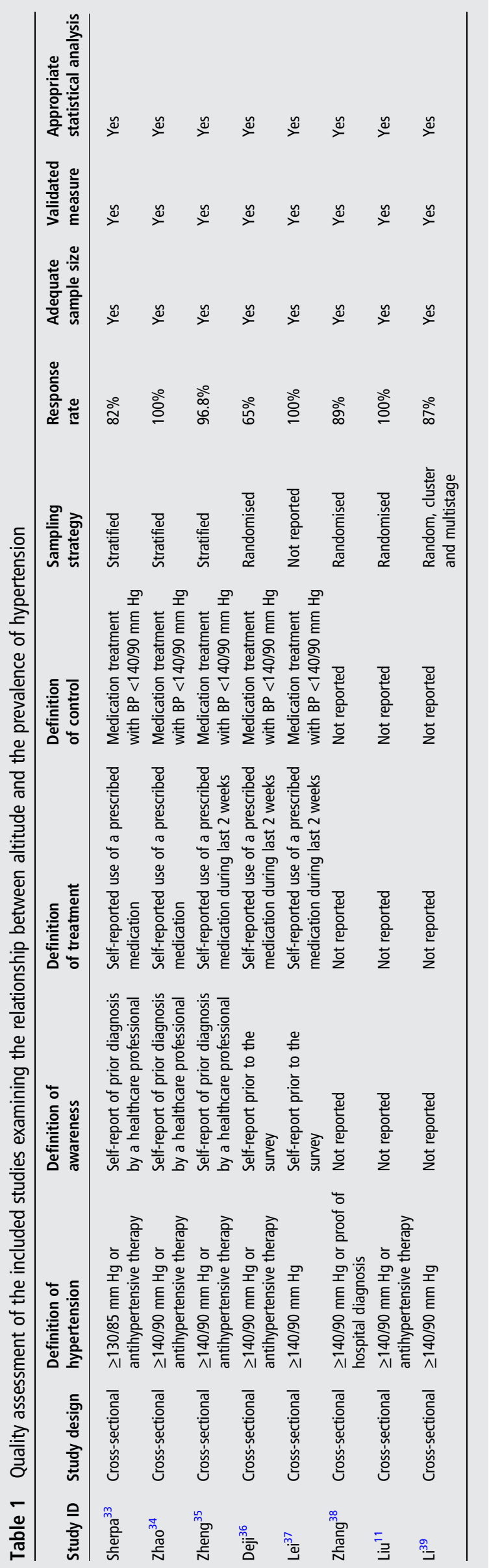




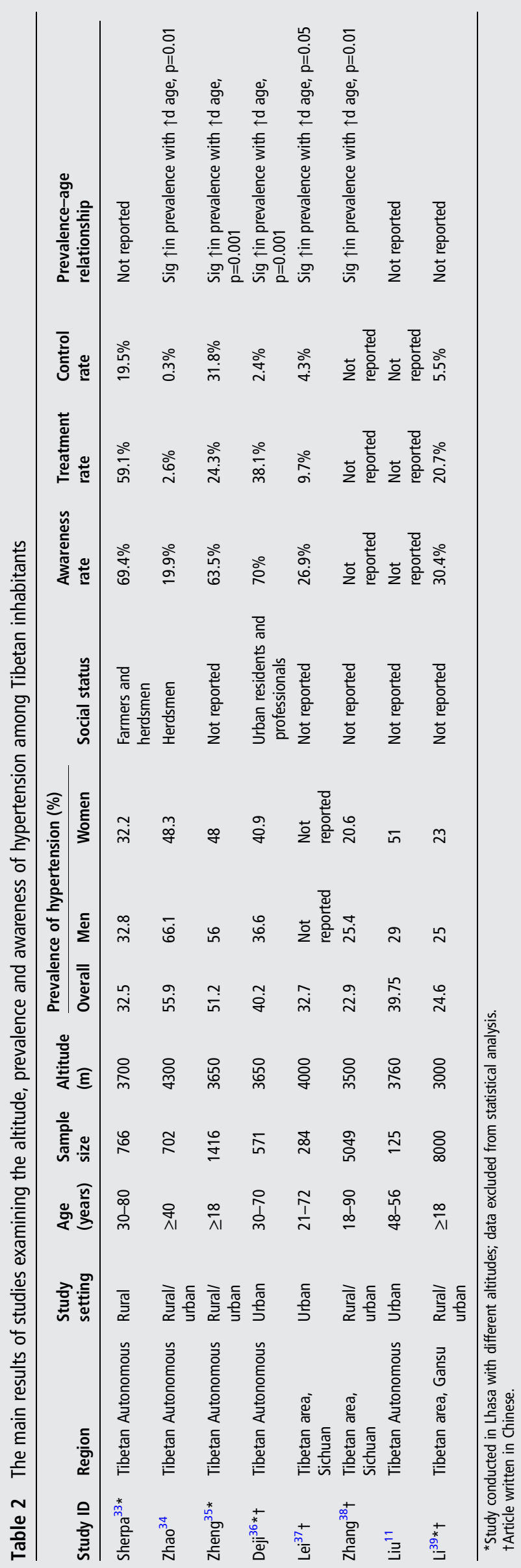

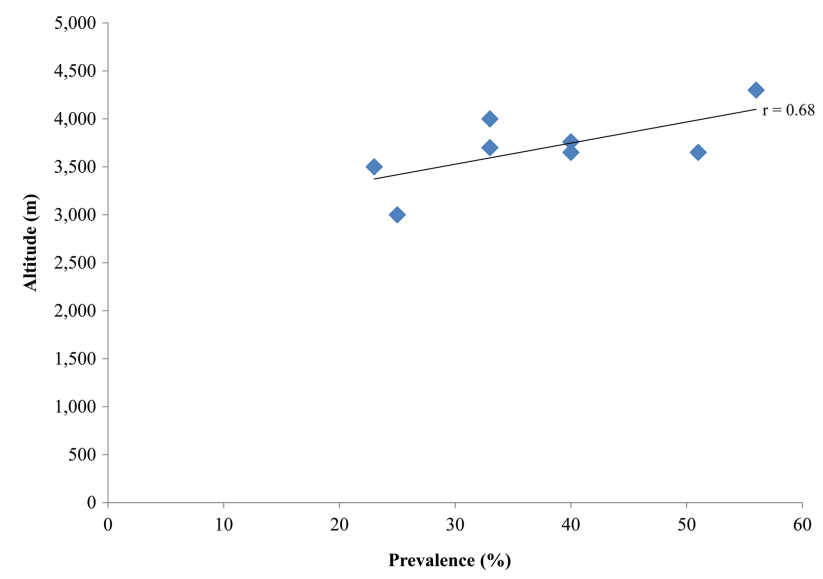

Figure 2 Relationship between altitude and the prevalence of hypertension. The $p$ value of the relationship was 0.04 .

Five studies reported data on the association between age and hypertension. All reported a significant increase in prevalence with increasing age: two reported $p$ values $<0.001,3536$ another two reported $\mathrm{p}<0.01 ;{ }^{34}{ }^{38}$ while one reported $\mathrm{p}<0.05 .{ }^{37}$ One study $^{35}$ reported significantly higher hypertension prevalence rates for urban, suburban and agricultural areas compared with pastoral areas $(\mathrm{p}<0.001)$; no other study compared prevalence rates between urban and rural settings.

Of the studies that reported hypertension prevalence according to gender, the prevalence of hypertension between male and female subjects differed over locations and age ranges, with prevalence ranging from $25.0 \%$ to $66.10 \%$ for men and from $20.6 \%$ to $51.0 \%$ for women. Three studies showed women as having greater hypertension prevalence, while the other five studies indicated men as having a higher prevalence (table 2). However, the differences in most studies were not apparent, while the prevalence result was noticeably higher in the female group at $51 \%$ versus male group at $29 \%$ in one particular study conducted in Lhasa. ${ }^{11}$ Separate scatter plots of altitude against prevalence for men and women did not reveal significant correlations between altitude and the prevalence of hypertension $(\mathrm{p}=0.13$ and $\mathrm{p}=0.18$, respectively), suggesting that gender may not have an influence on the prevalence of hypertension at high altitude.

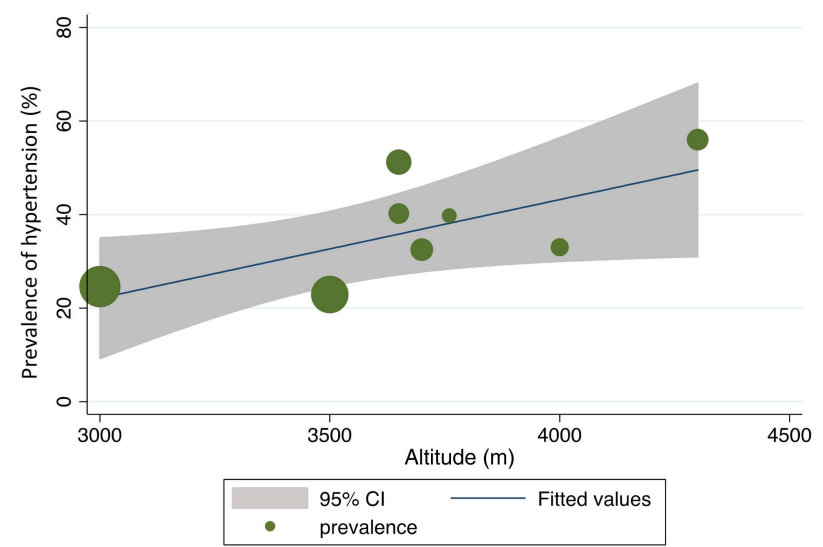

Figure 3 Meta-regression analysis showing the relationship between altitude and prevalence. The sizes of the circles correspond to the sample sizes of the included studies. There was a near significant relationship between altitude and prevalence $(p=0.06)$. An increase of $1 \mathrm{~m}$ in altitude was associated with a $0.02 \%$ increase in the prevalence of hypertension. 


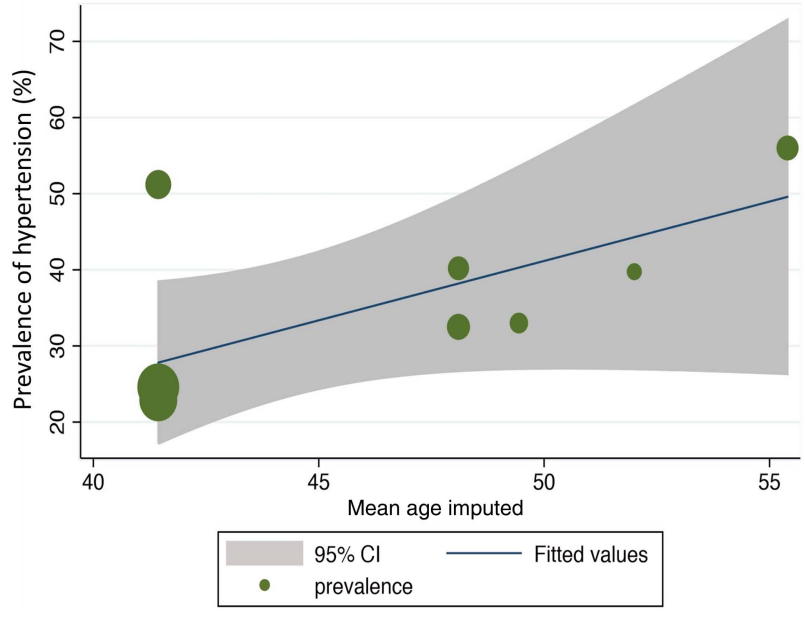

Figure 4 Meta-regression analysis showing the association between altitude, mean age and the prevalence of hypertension. There was a trend towards increased prevalence of hypertension with increasing altitude irrespective of age $(p=0.06)$. The meta-regression was based on imputed mean age using either the one given or imputing from a study that had similar age range (in one case using the median age for the age range reported). It was not possible to use age as a confounder due to inadequate data. The sizes of the circles correspond to the sample sizes of the included studies.

Six studies reported on the rates of awareness, treatment and control of hypertension. ${ }^{33-37} 39$ The highest rate of awareness was $70 \%$ in the City of Lhasa, where the socioeconomic status of subjects is at the level of urban residents/professionals, ${ }^{35}$ with the lowest rate being 20\% in Yangbajing Town, Dangxiong County, Lhasa Municipality, where the socioeconomic level is that of herdsmen. ${ }^{33}$ The highest treatment rate (59\%) was in rural Lhasa, ${ }^{32}$ with the lowest treatment rate reported at Yangbajing Town (2.6\%). ${ }^{33}$ Lhasa also had the highest control rate $(31.8 \%) ;{ }^{34}$ this study included data collected from both the city and nearby rural areas. The lowest control rate $(0.3 \%)$ was reported in Yangbajing Town. ${ }^{33}$ Apart from the studies conducted in Lhundub and Qushu Counties of Lhasa, all the other studies conducted in rural areas showed lower awareness, treatment and control rates. ${ }^{33} 3639$

\section{DISCUSSION}

Our results indicate that there is a significant correlation between altitude and the prevalence of hypertension among inhabitants of Tibet, corresponding to a $2 \%$ increase in prevalence for every $100 \mathrm{~m}$ increase in altitude. The results of our review also show that the locations and socioeconomic status of subjects affect the awareness and subsequent treatment and control of hypertension. The results from five of the included studies corroborate the findings from previous research indicating an age-related increase in the prevalence of hypertension in Tibet. ${ }^{40}$ Our results are also consistent with the findings of a recent randomised clinical trial which reported progressive increases in both conventional and ambulatory BPs with increasing altitude. ${ }^{41}$ To our knowledge, this is the first systematic review which examines the relationship between altitude and the prevalence of hypertension among Tibetan inhabitants.

The evidence of the impact of altitude on BP is controversial. $^{42}$ Researchers have previously attributed high altitude as a possible contributor to hypertension, and have suggested conducting of in-depth studies examining the correlation between altitude and $\mathrm{BP}^{43}$ It has been suggested that chronic exposure to high altitude results in increased sympathetic and parasympathetic activities, which lead to raised $\mathrm{BP}^{44}{ }^{45}$ However, there is very little research investigating the relationship between genetic adaptations and the prevalence of hypertension among Tibetan populations. According to $\mathrm{Li}$ and colleagues, ${ }^{8}$ two regulatory genes, the C allele at rs2070744 of the NOS3 and the T allele of rs4961 of the ADD1 gene, are significantly associated with the high prevalence of hypertension among Tibetan patients. Of these, the NOS3 gene was indicated as having a role in regulating vascular tone and blood vessel diameter, which may be altered by the low-oxygen environment of Tibet.

Though we found no differences in dietary lifestyle across the Tibetan regions, the influence of this variable on the prevalence of hypertension cannot be overlooked. Tibetans have been reported to consume 'traditional salty butter tea' whose salt content is four to five times the amount recommended by the WHO. ${ }^{7}{ }^{46}$ Indeed, the result of a recent study showed that a low-sodium, high-potassium salt-substitute intervention caused a dramatic fall in BP in hypertensive Tibetans. ${ }^{47}$

Even with the results of many studies illustrating that over time Tibetans can develop a certain degree of adaptation ability, such as greater forced vital capacity, lung diffusing capacity and blood haemoglobin concentration compared with lowland inhabitants, ${ }^{48}$ this does not necessarily imply that Tibetans do not experience hypoxia environmental challenge. The reduction of partial pressure gradients makes gas exchange more difficult, resulting in a long-term chronic insufficiency in the oxygenated blood circulation. This causes a drop in blood supply to the kidneys, and the subsequent release of renin that contributes to the vasoconstriction of the arteries. ${ }^{49}$

In terms of the influence of socioeconomic status on the awareness, treatment and control rates of hypertension, it is difficult to ascertain whether this is due to individuals' better education or due to levels of accessibility to local healthcare services, and thus further studies are required due to an absence of data. The contradictory results demonstrated by the study conducted in Lhasa Municipality, which reported the highest awareness and treatment rates $(70 \%$ and $38 \%)$, but with a very low control rate (2\%) may indicate the stress impact of hypertension in urban dwellers; this has been reported by other authors. ${ }^{50}$ Apart from the studies conducted in Lhundub and Qushu Counties, Lhasa reported a highest treatment rate of $59 \%$, and all other studies conducted in rural areas showed lower awareness, treatment and control rates. This may suggest that better health services are available in Lhundub and Qushu than in Yangbajing, due to distance and better transport facilities. The higher prevalence rates among herdsmen compared with multi-professionals in the Tibetan Autonomous regions (56\% vs $40 \%)$ illustrate the 'Inverse Care Law' where the availability of medical care inversely varies with the population served. ${ }^{51}$

The differences in the prevalence of hypertension between genders were minor and no particular trend emerged. A higher prevalence was found among women compared with men in one study. ${ }^{11}$ However, in another study with similar participant characteristics (albeit with a larger sample size), ${ }^{34}$ the reverse was the case. Further research investigating whether gender is a risk factor for hypertension at high altitude will help remove this uncertainty.

\section{Strengths and limitations}

This review has some strengths. It is the first which synthesises the information on the relationship between altitude and the prevalence of hypertension in Tibet. We employed a robust search strategy, and the included studies were generally of good 
reporting quality. The consistency observed in most of the relationships explored also suggests that altitude has a role in the pathogenesis of hypertension among Tibetans. However, we recognise some limitations. The included studies were all crosssectional, and therefore not ideal for establishing causality. The review results are also only applicable to Tibetan populations. We also may not have identified all studies examining the relationship between altitude and the prevalence of hypertension among inhabitants of Tibet. In addition, the results could have been confounded by the variations in age, non-response bias and inconsistencies in awareness, treatment and control. Furthermore, the reporting of the data was not ideal; therefore, individual patient data would better have allowed us to explore the relationship between altitude and BP adjusted for other important confounders such as age and sex.

\section{Implications for research and practice}

Most published surveys conducted so far are mainly concentrated in Lhasa, the capital city of Tibet, and surrounding counties, and rarely has research been carried out in other prefectures of Tibet where the altitude is much higher $(\geq 4500 \mathrm{~m})$ and living conditions harsher. Thus, more health surveys would provide an in-depth view of the relationship between high altitude and hypertension. In addition, further research is needed to identify the underlying mechanisms of why exposure to high altitude could lead to variable results. To achieve this, it may be important to consider the impact of altitude on hypertension in relation to its variable pathological states to identify an in-depth causality which is applicable to wider populations.

The significant correlation between altitude and the prevalence of hypertension in our review could help to identify high altitude as an important cause of hypertension at high altitude. This finding may provide new insights into the prevention and treatment of hypertension at high altitudes, resulting in a reduction of subsequent cardiovascular problems (eg, stroke and ischaemic heart disease), thereby increasing the overall life expectancy of high-altitude dwellers; indeed, research evidence

Key messages

What is already known on this subject?

- The results of genetic and observational research have shown that Tibet has a higher hypertension rate than in other Chinese areas.

- There is uncertainty about the relationship between high altitude and hypertension, and various hypotheses have been proposed.

\section{What might this study add?}

- There is a statistically significant correlation between altitude and the prevalence of hypertension in Tibet.

- An increase of $100 \mathrm{~m}$ in altitude is associated with a $2 \%$ increase in the prevalence of hypertension among Tibetan inhabitants.

- Socioeconomic status may influence the rates of awareness and subsequent control and treatment of hypertension.

\section{How might this impact on clinical practice?}

- Living at high altitudes could be a potential risk factor for hypertension. suggests that commonly used BP medications may be ineffective at high altitudes. ${ }^{41}$ In addition, better understanding of the altitude-hypertension relationship may help provide novel techniques for managing conditions such as hypoxia of obstructive sleep apnoea which mimic some aspects of altitude.

\section{CONCLUSION}

The results from cross-sectional studies suggest that there is a significant correlation between altitude and the prevalence of hypertension among inhabitants of Tibet. The results of our review also show that the locations and socioeconomic status of subjects can influence awareness and subsequent treatment and control of hypertension. Very little research into hypertension has been conducted in other prefectures of Tibet where the altitude is much higher. Further research examining the impact of altitude on BP among Tibetan inhabitants is warranted.

Acknowledgements We would like to thank Nia Roberts for help with conducting the electronic searches, and Liu Yashi for assistance with translation of Chinese articles.

Contributors CM and IJO were involved with data extraction, data analysis and interpretation and co-drafting of the manuscript. RP was involved with data analysis and interpretation of the manuscript. AMW was involved with data interpretation and co-drafting of the manuscript. CJH was involved with data analysis and interpretation, and co-drafting of the manuscript.

Funding This study was funded by The Wellcome Trust, UK (Wellcome Trust grant reference number: 095772/Z/11/Z). The funders played no role in any aspect of the preparation of the manuscript.

Competing interests $\mathrm{CJH}$ receives payment for running educational courses at the University of Oxford and University of Oxford ISIS Consulting Services for external teaching and training. He also receives royalties for books (Evidence Based Toolkit series by Blackwell BMJ Books). CM, IJO, RP and AMW have no interests to disclose.

Provenance and peer review Not commissioned; externally peer reviewed.

Open Access This is an Open Access article distributed in accordance with the terms of the Creative Commons Attribution (CC BY 4.0) license, which permits others to distribute, remix, adapt and build upon this work, for commercial use, provided the original work is properly cited. See: http://creativecommons.org/ licenses/by/4.0/

\section{REFERENCES}

1 Institute of Medicine (US) Committee on Preventing the Global Epidemic of Cardiovascular Disease: Meeting the Challenges in Developing Countries. In: Fuster V, Kelly BB, eds. Promoting Global Cardiovascular Health in the Developing World: A Critical Challenge to Achieve Global Health. Washington DC: National Academies Press (US), 2010. The National Academies Collection: Reports funded by National Institutes of Health www.ncbi.n/m.nih.gov/pubmed/20945571.

2 Brito J, Siqués $P$, León-Velarde $F$, et al. Chronic intermittent hypoxia at high altitude exposure for over 12 years: assessment of hematological, cardiovascular, and renal effects. High Alt Med Biol 2007;8:236-44.

3 Wolfel EE, Selland MA, Mazzeo RS, et al. Systemic hypertension at 4,300 m is related to sympathoadrenal activity. J Appl Physiol (1985) 1994;76:1643-50.

4 Handler J. Altitude-related hypertension. J Clin Hypertens (Greenwich) 2009:11:161-5.

5 China.org.cn. The Tibetan ethnic minority. http://www.china.org.cn/e-groups/ shaoshu/shao-2-tibetan.htm (accessed 17 Mar 2014).

6 China Demographics Profile. http://www.indexmundi.com/china/demographics_ profile.html (accessed 15 Jan 2014).

7 Sun SF. Epidemiology of hypertension on the Tibetan Plateau. Hum Biol 1986;58:507-15

8 Li K, Liang Y, Sun Y, et al. The relationship between polymorphisms at 17 gene sites and hypertension among the aboriginal Tibetan people. Biomed Environ Sci 2012;25:526-32.

9 Monge MC, Pesce H. El sistema nervioso vegetativo del hombre de los Andes. Ann Fac Cienc Med 1935:17:43-59.

$10 \mathrm{Hu}$ YS, Yao CH, Wang WZ, et al. [Survey on the prevalence of hypertension in different ethnic groups in China in 2002]. Wei Sheng Yen Chiu 2006:35:573-5

11 Liu L, Liu L, Ding Y, et al. Ethnic and environmental differences in various markers of dietary intake and blood pressure among Chinese Han and three other minority peoples of China: results from the WHO Cardiovascular Diseases and Alimentary Comparison (CARDIAC) Study. Hypertens Res 2001;24:315-22. 
12 Clegg EJ, Jeffries DJ, Harrison GA. Determinants of blood pressure at high and low altitudes in Ethiopia. Proc $R$ Soc Lond B 1976;194:63-81.

13 Frisancho AR. Human adaption: a functional interpretation. St. Louis, MO: C. V. Mosby, 1979.

14 Somers VK, Mark AL, Abboud FM. Potentiation of symphathetic nerve responses to hypoxia in borderline hypertensive subjects. Hypertension 1988;11:608-12.

15 Hanna JM. Climate, altitude, and blood pressure. Hum Biol 1999;71:553-82.

16 Wu TY, Ding SQ, Liu JL, et al. Who should not go high: chronic disease and work at altitude during construction of the Qinghai-Tibet railroad. High Alt Med Biol 2007:8:88-107.

17 Marticorena E, Ruiz L, Severino J, et al. Systemic blood pressure in white men born at sea level: changes after long residence at high altitudes. Am J Cardiol 1969:23:364-8.

18 Ruiz L, Peñaloza D. Altitude and hypertension. Mayo Clin Proc 1977;52:442-5.

19 Mirrakhimov MM. Biological and physiological characteristics of the high altitude natives of Tien Shan and Pamirs. In: Mirrakhimov MM, ed. The biology of high altitude peoples. New York: Cambridge University Press, 1978:299-316.

20 Moore LG, Hershey DW, Jahnigen D, et al. The incidence of pregnancy-induced hypertension is increased among Colorado residents at high altitude. Am J Obstet Gynecol 1982;144:423-9.

21 Sun S. Review of physiological survey of Tibetan plateau over past two years. Tibetan Med 1978;5:1-8

22 von Elm E, Altman DG, Egger M, et al. The Strengthening the Reporting of Observational Studies in Epidemiology (STROBE) statement: guidelines for reporting observational studies. PLoS Med 2007;4:e296.

23 Dawaciren, Gesangluobu, Zhuomaciren, et al. [Association between degree of pulmonary artery hypertension and cardiac structural and functional changes in chronic high altitude heart disease patients]. [Article in Chinese]. Zhonghua Xin Xue Guan Bing Za Zhi 2013;41:761-5.

24 Buroker NE, Ning XH, Zhou ZN, et al. Genetic associations with mountain sickness in Han and Tibetan residents at the Qinghai-Tibetan Plateau. Clin Chim Acta 2010;411:1466-73

25 Lin J, Zheng B, Li S, et al. Dysarteriotony state of officers and soldiers stationed at above altitude of $4500 \mathrm{~m}$ from a unit in Northern Tibet. Occup Health 2012;28:672-4.

26 Pei $T$, Li X, Tao F, et al. Burden of disease resulting from chronic mountain sickness among young Chinese male immigrants in Tibet. BMC Public Health 2012;12:401.

27 Bera S. A study on blood pressures between the Tibet born and India born Tibetans who are permanently residing in Northern India. Coll Antropol 2006:30:749-52.

28 Yuan Z, Wang $\mathrm{X}$, Hou M. Changes in blood pressure, electrocardiogram and cardio-thoracic ratio of people returned to plain from plateau. $J$ Environ Health 2010;27:987-90.

29 Hurtado A, Escudero E, Pando J, et al. Cardiovascular and renal effects of chronic exposure to high altitude. Nephrol Dial Transplant 2012;27:iv11-16.

30 [Prevalence rates and risk factors of metabolic syndrome in Tibetans]. [Article in Chinese]. Chin J Med 2009;9:121-2.
31 Zhao X, Li S, Li N, et al. Prevalence, awareness, treatment, and control of hypertension in Yangbajing, an area at an altitude of over 4000 meters above sea level in Tibet. Circulation 2010;122:e77-8.

32 Yang S, Liu S, Wang Y, et al. High blood pressure in Chinese ethnic minorities: report from rural Yunnan province. Am J Hypertens 2011;24:1209-14.

33 Sherpa LY, Deji, Stigum H, et al. Prevalence of metabolic syndrome and common metabolic components in high altitude farmers and herdsmen at $3700 \mathrm{~m}$ in Tibet. High Alt Med Biol 2013;14:37-44.

34 Zhao X, Li S, Ba S, et al. Prevalence, awareness, treatment, and control of hypertension among herdsmen living at 4,300 $\mathrm{m}$ in Tibet. Am J Hypertens 2012;25:583-9.

35 Zheng $X$, Yao DK, Zhuo-Ma CR, et al. Prevalence, self-awareness, treatment, and control of hypertension in Lhasa, Tibet. Clin Exp Hypertens (New York) 2012;34:328-33.

36 Deji, Dawapuchi, Danzeng, et al. [Prevalence of hypertension among 30 to 70 years old citizens of Lhasa, Tibet China]. [Article in Chinese]. Zhonghua Xin Xue Guan Bing Za Zhi 2010;38:755-8.

37 Lei $Y$, Hong Z, Hongbin T, et al. Comparison of prevalence of hypertension and abnormal blood lipoid between Tibetans living at high altitude and Han people living in plain. Med J Nat Defending Forces Southwest China 2010;20:989-91.

38 Zhang HT, Zhou YL, Gao L, et al. An epidemiological study on the hypertension and cerebral stroke in Ganzi Tibetan state. Chin J Evid Based Med 2012;12:173-5.

39 Li XH, Qiao Y, Li BJ, et al. [Prevalence and awareness of hypertension in Gansu Gannan Tibetan adult population]. [Article in Chinese]. Zhonghua Xin Xue Guan Bing Za Zhi 2012;40:527-9.

40 Tripathy V, Gupta R. Blood pressure variation among Tibetans at different altitudes. Ann Hum Biol 2007;34:470-83.

41 Parati G, Bilo G, Faini A, et al. Changes in $24 \mathrm{~h}$ ambulatory blood pressure and effects of angiotensin II receptor blockade during acute and prolonged high-altitude exposure: a randomized clinical trial. Eur Heart J 2014;35:3113-22.

42 Khalid MEH. The effect of altitude on blood pressure in the Assir Province of Saudi Arabia. Wilderness Environ Med 1995;6:401-6.

43 Sizlan A, Ogur R, Ozer $M$, et al. Blood pressure changes in young male subjects exposed to a median altitude. Clin Auton Res 2008;18:84-9.

44 Bärtsch P, Gibbs JS. Effect of altitude on the heart and the lungs. Circulation 2007;116:2191-202.

45 Liu L, Mizushima S, Gao M, et al. Body mass index, urinary sodium excretion, and blood pressure in seven Chinese populations: results from the WHO Cardiovascular Diseases and Alimentary Comparison Study. CVD Prev 2000;3:11-17.

46 Sehgal AK, Krishan I, Malhotra RP, et al. Observations on the blood pressure of Tibetans. Circulation 1968;37:36-44.

47 Zhao $X$, Yin $X$, Li X, et al. Using a low-sodium, high-potassium salt substitute to reduce blood pressure among Tibetans with high blood pressure: a patient-blinded randomized controlled trial. PLOS ONE 2014;9:e110131.

48 Wu T, Kayser B. High altitude adaptation in Tibetans. High Alt Med Biol 2006; 7:193-208.

49 Howden R, Kleeberger SR. Genetic and environmental influences on gas exchange. Compr Physiol 2012;2:2595-614.

50 Faye K, Heng LH, Collomp R, et al. Hypertension and stress. J Mal Vasc 2003;28:4-8.

51 Hart JT. The inverse care law. Lancet 1971;297:405-12. 